\title{
MAGNETO-OPTICAL FARADAY EFFECT OF COBALT NANO-CLUSTERS ORIENTED UNIAXIALLY IN A TRANSPARENT PLASTIC
}

\author{
M. Inoue ${ }^{1}$, T. Yamamoto ${ }^{1}$, K. Isamoto ${ }^{1}$, A. Horikawa ${ }^{1}$, and T. Fujiil ${ }^{1 \& 2}$

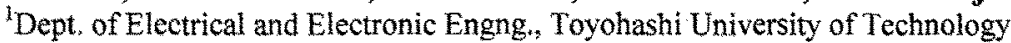 \\ 1-1 Hibari-Ga-Oka, Tempaku, Toyohashi 441, Japan \\ ${ }^{2}$ Reserch Institute of Electrical Communication. Tohoku University, Sendai 980-77, Japan
}

\begin{abstract}
Magneto-optical Faraday effect of cobalt nano-clusters oriented uniaxially in a transparent matrix was studied. The experimental results showed that the oriented films were good polarizing plates with very unique MO properties which varied depending on the geometry of fim structures and the polarization of incident lights. Numerical analysis revealed that the MO properties originated in the mode conversion of lights from TE to TM, arising from the magnetic and optical uniaxial anisotropies.
\end{abstract}

KEYWORDS: MAGNETO-OPTICAL FARADAY EFFECT, NANO-CLUSTERS, DISCRETE FILMS

\section{INTRODUCTION}

Within the past several years, a growing interest in magneto-optical (MO) effect of composite magnetic films with ferromagnetic fine particles dispersed in transparent matrices has resulted from their unique MO properties. In fact, our successive studies of the discrete magnetic films showed that the MO Faraday spectra (MO-FS shortly) of these films were subject to considerable variations depending on the concentration and aggregation states of the ferromagnetic fine particles, where substantial enhancement in their Faraday rotation angles and/or systematic change in their shapes were observed [1], [2]. These results imply that the introduction and the arrangement of discrete structures in magnetic films lead to an artificial design of the MO properties, which is, indeed, very attractive for various optoelectronic applications.

An effective method for actively controlling the MO effect is a use of discrete films with ferromagnetic fine clusters which are oriented uniaxially in a transparent matrix. This is because the uniaxial orientation of the magnetic clusters yields magnetic and optical uniaxial anisotropies in the films, and their MO properties can be controlled by arranging the magnitude and the direction of the anisotropies. In this respect, we studied experimentally and theoretically the MO properties of cobalt films with uniaxially oriented discrete structures. The oriented films were found to be good polarizing plates with very unique MO properties; their MOFS varied considerably depending on geometry of the film structures and the polarization of incident lights. Numerical analysis revealed that the MO properties originated in the mode conversion of lights from TE to TM, arising from the magnetic and optical uniaxial anisotropies.

\section{FILM PREPARATION}

Cobalt fine particles with fec crystallographic structure were prepared by thermally decomposing dicobalt octacarbonyl $\left[\mathrm{CO}_{2}(\mathrm{CO})_{8}\right.$, shortly DO] which was dissolved in toluene solution of polymethyl methacrylate (PMMA, hereafter.) The thermal decomposition of the DO liquid was achieved at $110^{\circ} \mathrm{C}$ for more than 6 hours by stirring the liquid in a nitrogen flow. The colloidal solution thus obtained was spin- coated on a glass substrate and dried at room temperature. Toluene in the solution was evacuated during the solidification process, and a discrete film, in which cobalt fine particles were dispersed in PMMA, was obtained.

To express the cobalt particle concentration in PMMA, we used the weight ratio of DO to PMMA defined by $C_{c 0}=$ $W_{D O} /\left(W_{D O}+W_{P M M A}\right)$. A typical TEM picture of the cobalt particles dispersed in PMMA is shown in Fig. 1 for the case of $C_{c o}=33.3 \%$. The average diameter of the cobalt particles was $10 \mathrm{~nm}$ from this figure, and was found to be controllable by the value of $C_{c o}$.

When a do magnetic field is applied during the film solidification process, filament-like cobalt clusters elongating along the field were formed in the films. The cluster configuration was varied by the field strength and the viscosity of the solution. Figure 2 shows a microscope picture of the uniaxially oriented discrete film which was obtained from the solution with $C_{c o}=75 \%$ and $C_{f p}=75 \%$ under the filed strength of $2000 \mathrm{e}$. Here, $C_{f p}$ is another index expressing the cobalt con-

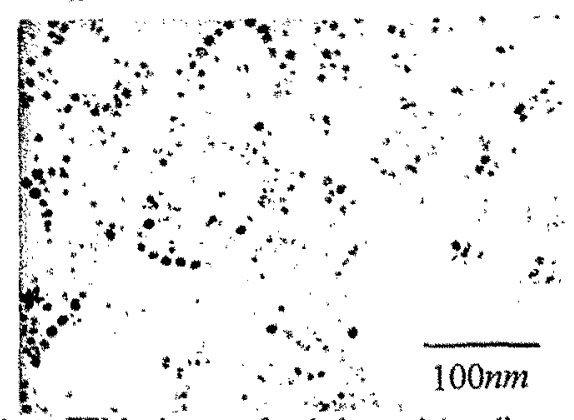

Fig.1 A TEM picture of cobalt particles dispersed in PMMA, where $C_{c a}=33.3 \%$

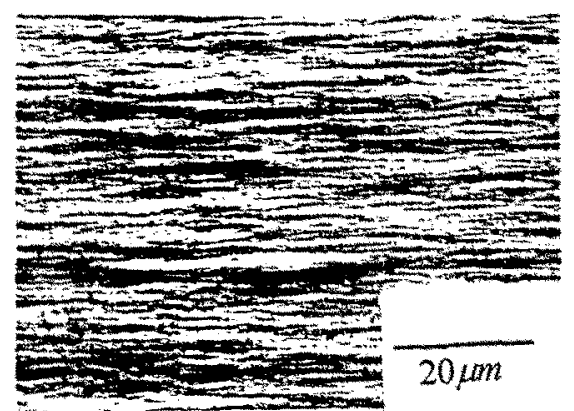

Fig. 2 A microscope picture of uniaxially oriented $\mathrm{Co}$ cluster film, where $C_{c 0}=75 \%, C_{f p}=60 \%$ and $H d=2000$ e. 
centration of the solution, which is defined by $C_{f p}=$ $W_{D O} /\left(W_{D O}+W_{P M M A}+W_{P M M A}\right)$ with the weight of additional PMMA $W^{\prime}{ }_{P M M A}$ supplemented to the solution after the thermal decomposition. Note that the value of $C_{f f}$ represents the dilution degree of the solution, in which the diameter of cobalt particles is governed by the value of $C_{c o}$.

\section{MO PROPERTIES OF THE FILMS}

The discrete films prepared with a magnetic field had uniaxial inplane magnetic anisotropies whose anisotropy energy was estimated to be $K u \sim 10^{6} \mathrm{erg} / \mathrm{cm}^{3}$. The uniaxial orientation of the cobalt clusters also induced a uniaxial optical anisotropy: In Fig.3, the optical transmittance characteristics of the films prepared at $H_{d}=0 \mathrm{Oe}$ (marked by $\bullet$ ) and $H_{d}=3 \mathrm{kOe}$ (marked by $\mathrm{O}$ ) are shown as a function of the angle $\theta$ measured from the cluster axis. The measurement was made using a linearly polarized light with $\lambda=633 \mathrm{~nm}$. The oriented film acted as a good polarizing plate while no apparent change in its transmittance was observed for the non-oriented film.

Figures 4 and 5 show the MO-FS (Faraday rotation angle $\theta_{F}$ and ellipticity $\eta_{F}$ versus wavelength $\lambda$ ) of the cobalt discrete films prepared without applying a magnetic field. In Fig.4, the films were those of $C_{c o}=75 \%$ (the average diameter of cobalt particles was about $20 \mathrm{~nm}$ ) and $C_{f p}=40 \sim 60 \%$. The change in $C_{f p}$ leads to a variation in the magnitudes of spectra without causing a significant change in their shapes. On the other hand, in Fig.5, films were those of low concentration of cobalt particles $\left(C_{c o}=10 \sim 30 \%\right)$ whose diameters ranged from $5 \mathrm{~nm}$ to $10 \mathrm{~nm}$. In this case, both $\theta_{F}$ and $\eta_{F}$ are about $10 \%$ of those in Fig.4, because of small magnetizations due to the small sizes of cobalt particles.

MO-FS of the uniaxially oriented films are shown in Figs. 6 and 7, where Fig.6 is the case of TM light incidence ( $\theta=0$ in Fig.3) while Fig.7 is the case of TE light incidence ( $\theta=90$ in Fig.3.) In Fig.6, both $\theta_{F}$ and $\eta_{F}$ are very small in comparison with those in Fig.7, suggesting that the MO effect for TM light incidence vanishes. This is because, as explained in the subsequent analysis, the TM light submerged in the medium propagates in the PMMA region exclusively. As for TE light incidence in Fig.7, however, $\theta_{F}$ and $\eta_{F}$ are about two times larger than those in Fig.4, showing the uniaxial orientation of cobalt clusters enhances the MO effect considerably. It is interesting in this case that the change in $C_{f p}$

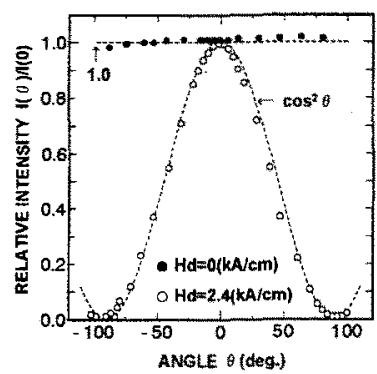

Fig. 3 Optical transmittance of the films at $633 \mathrm{~nm}$, where $\theta$ was taken from the cluster axis.
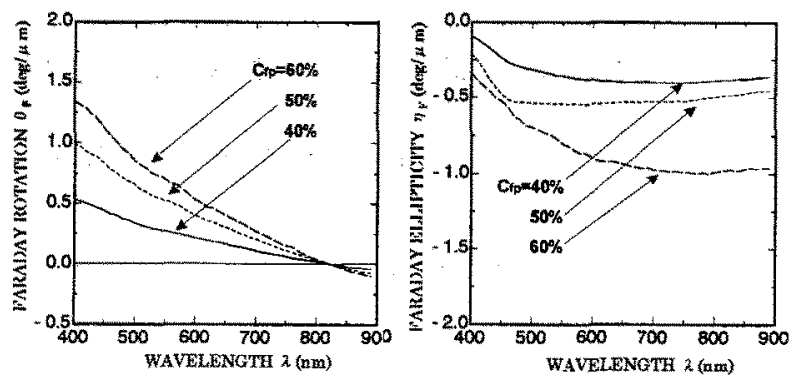

Fig. 4 MO-FS of the cobalt discrete films of $C_{c o}=75 \%$ and $C_{f p}=40 \sim 60 \%$ prepared without a magnetic field.
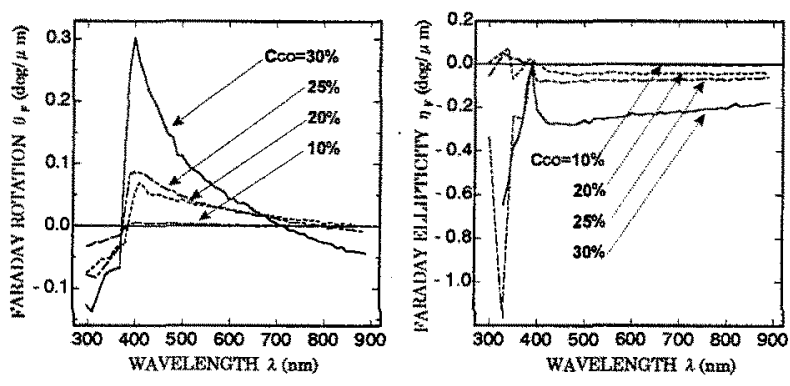

Fig. 5 MO-FS of the cobalt discrete films of $C_{c o}=10-30 \%$ prepared without a magnetic field.
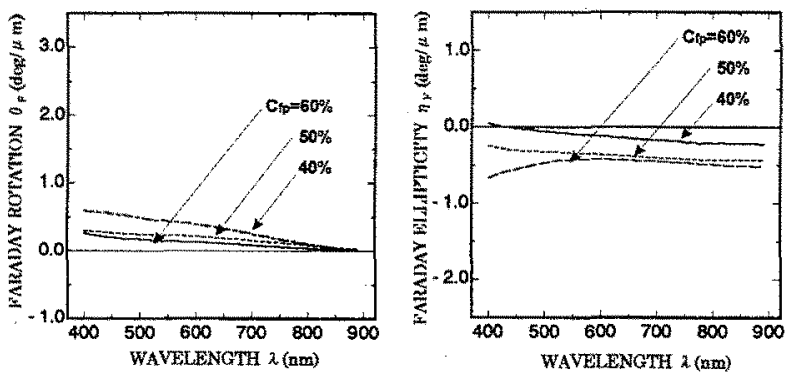

Fig. $6 \mathrm{MO}-\mathrm{FS}$ of the uniaxially oriented films of $C_{c o}=75 \%$ and $C_{f f}=40 \sim 60 \%$ for the case of TM light incidence.
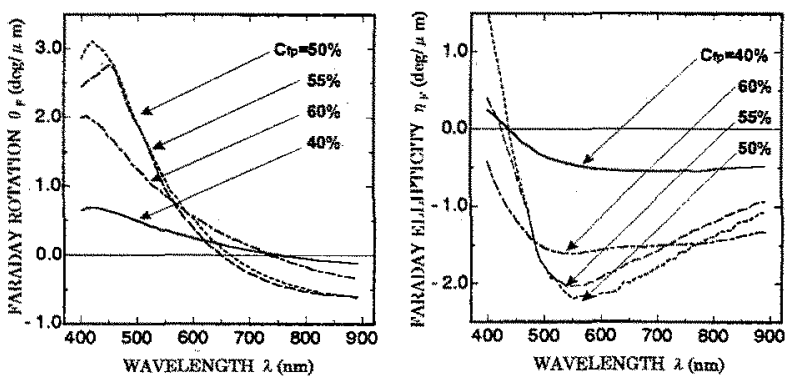

Fig. 7 MO-FS of the films in Fig. 6 of for the case of TE light incidence.

value causes the variations not only in the magnitudes of $\mathrm{MO}$ FS but in their shapes (see the zero-cross wavelength, for instance), although such a change in the MO-FS shapes was not seen apparently in the non-oriented films in Fig.4.

\section{THEORETICAL ANALYSIS}

An ideal discrete medium with one-dimensional quasirandom array structure is considered [3]: As shown in Figs. 8(a) and $8(b)$, the medium comprises of a periodic array of equivalent virtual segment with width $D$ containing $N$ subseg- 
ments of either cobalt or air-gap instead of PMMA for simplicity. The widths of the cobalt and air-gap subsegments are, respectively, $d_{l}$ and $d_{0}$, which are connected by the relation of $D=N_{l} d_{l}+\left(N-N_{l}\right) d_{0}$ with the number of cobalt subsegments $N_{l}$. The sequence of the subsegments was random designed with a computer-generated random binary number $b_{N}$ of $N$ digits corresponding to each subsegment, where cobalt (air-gap) subsegment is assigned if the digit is " 1 " ( 0 ".) Then, the packing density of cobalt in the medium is defined by $P_{M}=N_{l} d_{l} / D$.

The theoretical approaches [4] are summarized as follows: To obtain MO-FS of the media, exact values of complex refractive indices of lights, $n^{\prime}=n+i \kappa$, were first determined numerically from the Maxwell equations (1) and (2):

$$
\begin{aligned}
& \nabla \times \mathbf{E}=-\mu_{0} \partial \mathbf{H} / \partial t \\
& \nabla \times \mathbf{H}=\varepsilon_{0} \widetilde{\varepsilon} \partial \mathbf{E} / \partial t
\end{aligned}
$$

These equations were solved using the random matrix approach under the complex boundary conditions imposed at every discontinuous surfaces. When the light has a plane wave form, Eqs. (1) and (2) are arranged into the following simultaneous differential equations:

$$
\mathrm{d} \mathbf{t}(Y) / \mathrm{d} Y=\mathbf{A}_{i}\left(n^{\prime}, \omega\right) \mathbf{t}(Y)
$$

where $\mathbf{A}_{i}\left(n^{\prime}, \omega\right)$ is the state matrix of the cobalt $(i=1)$ and airgap $(i=0)$ subsegments and $\mathrm{t}(Y)$ is the state vector defined by $\mathbf{t}(Y)=\left(E_{X}(Y), E_{Z}(Y), H_{X}(Y), H_{Z}(Y)\right)$. Then, for one virtual segment with width $D$, the state vectors at the boundary surfaces of $Y=0$ and $Y=D$ are connected from eq. (3) as

$$
\mathbf{t}(D)=\Phi_{1} \cdot \Phi_{1} \cdot \Phi_{0} \cdot \Phi_{1} \cdots \Phi_{0} \cdot \Phi_{1} \cdot \mathbf{t}(0)
$$

where $\Phi_{i}$ ( $i=0$ and 1) are the transition matrices defined by $\Phi_{i}=\exp \left[\mathbf{A}_{i} d_{i}\right]$ whose sequence corresponds to the sequence of " 1 " and " 0 " in $b_{N}$. The periodic translation symmetry of the medium imposes $t(D)=t(0)$, and thus from (4) we have

$$
\mathbf{B}\left(n^{\prime}, \omega\right) \cdot \mathbf{t}(0)=0
$$

where $\mathbf{B}\left(n^{\prime}, \omega\right)=\Phi_{1} \cdot \Phi_{1} \cdot \Phi_{0} \cdot \Phi_{1} \ldots \Phi_{0} \cdot \Phi_{1}-\mathbf{I}_{4}$ with a 4 $\times 4$ unit matrix $\mathbf{I}_{4}$. The refractive index $n^{\prime}$ is now obtained from Eq.(5) as a complex quantity satisfying det.B $=0$. This condition is, in general, satisfied by two values of $n^{\prime}$ corresponding to those of two fundamental eigenmodes of lights. For convenience, we hereafter call them as $\mathrm{R}$-mode and $\mathrm{L}$ mode lights, because the $\mathrm{R}(\mathrm{L})$-mode light degenerates into right(left)-hand circularly polarized light when $P_{M} \rightarrow 100 \%$ (continuous magnetic medium.)

The rotation angle $\theta(L)$ and the ellipticity $\eta(L)$ of light in the medium were evaluated as observable quantities practically by using the electric field expressed as a linear combination of the R-mode and L-mode of lights. In accor-

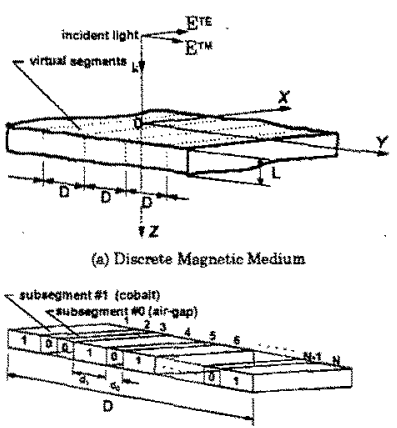

(b) Uniaxislly Criented Cobalt Subsegnent

Fig. 8 Model used for the calculations.

dance with a common experimental procedure, they are given as follows:

$$
\begin{aligned}
& \tan 2 \theta(L)=2 \int_{b}^{D} \operatorname{Re}\left\{E_{X}(Y, L) E_{Y}^{*}(Y, L)\right\} d Y \\
& \times\left\{\int_{0}^{D}\left|E_{Y}(Y, L)\right|^{2} d Y-\int_{0}^{D}\left|E_{X}(Y, L)\right|^{2} d Y\right\}^{-1}
\end{aligned}
$$

and

$$
\begin{aligned}
\tan 2 & \eta(L)=2 \int_{0}^{D} \operatorname{Re}\left\{i E_{X^{\prime}}(Y, L) E_{Y^{\prime}}^{*}(Y, L)\right\} d Y \\
\times & \left\{\int_{0}^{D}\left|E_{X^{\prime}}(Y, L)\right|^{2} d Y-\int_{0}^{D}\left|i E_{Y^{\prime}}(Y, L)\right|^{2} d Y\right\}^{-1}
\end{aligned}
$$

where we used a rotation of the coordinate system from $X$ and $Y$ axes to $X^{\prime}$ and $Y^{\prime}$ axes which are a new set of axes along the principal axes of the resultant electric field with elliptical polarization on the $X-Y$ plane.

\section{NUMERICAL RESULTS}

Numerical calculations were achieved using the dielectric elements of cobalt film whose values were determined from the magneto-optical quantities given in [5] and [6]. As for structural parameters, we used $D=50 \mathrm{~nm}, N=16, L$ (film thickness) $=0.3 \mu \mathrm{m}$ and $P_{M}=0 \sim 100 \%$.

The dispersion curves of the refractive indices of lights are shown in Fig.9(a) ( $n^{\prime}$ vs. $\lambda$ when $P_{M}=30 \%$ ) and Fig.9(b) ( $n$ 'vs. $P_{M}$ when $\lambda=381.5 \mathrm{~nm}$ ), where the superscripts $\mathrm{R}$ and $\mathrm{L}$ denote the R-mode and L-mode of lights, respectively. Near $P_{M}=100 \%$ in Fig.9(b), $n$ ' of both modes of lights exactly coincide with those of the continuous cobalt film. Except this highly packed limit, $\kappa^{2}$ is much smaller than $\kappa^{R}$ and their difference is enlarged with increasing the wavelength of light and/or decreasing the packing density, indicating that the $\mathrm{L}(\mathrm{R})$-mode of light is the transmission (rejection) mode of light. This is caused by the uniaxial anisotropy of the medium structure, where the L-mode light tends to be a linearly polarized TM light propagating mainly in the air-gaps, while the R-mode light becomes a linearly polarized TE light which propagates uniformly spreading in the medium. Figure 10(a) illustrates this situation clearly by showing the spatial distribution of power densities carried by the L-mode of light 


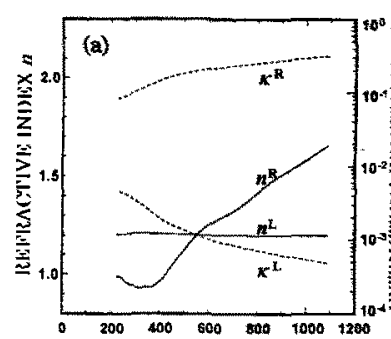

WAVELENGTH $\lambda[\mathrm{nm}]$

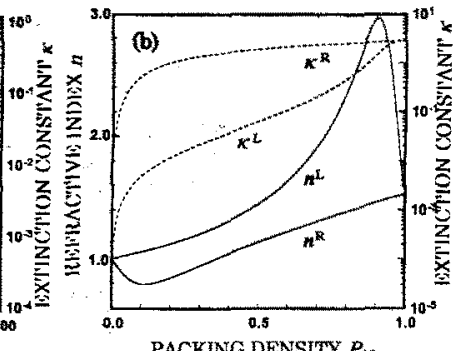

PACKING DENSITYY $P_{M}$
Fig. 9 Dispersion relations of the $R$ - and $L$ modes of lights in the medium, where $P_{M}=30 \%$ in (a) and $\lambda=381.5 \mathrm{~nm}$ in (b).

(TM light; broken line) and the R-mode of light (TE light; solid line) in the medium with $P_{M}=30 \%$. The L-mode light is indeed confined within the air-gaps whereas the R-mode light penetrates in the magnetic subsegments (light black stripes.) Therefore, when TM light is incident to the medium, the Lmode light is excited selectively, for which the contribution from the magnetic subsegments is considerably small. However, the incidence of TE light exclusively excites the R-mode light penetrating in the magnetic subsegments, and then the $\mathrm{MO}$ effect arises via interaction with magnetizations.

To clarify the MO effect of the medium with thickness $L$, we calculated the rotation angle $\theta(L)$ and the ellipticity $\eta(L)$ for TE and TM lights incidences. The typical result for the case of TE light incidence is shown in Fig.10(b): A stepwise change in $\theta(L)$ (90-degree rotation) takes place within the medium thickness less than $0.5 \mu \mathrm{m}$. This suggests that the light mode conversion from TE to TM lights occurs in the oriented discrete media, which arises from the uniaxial array of cobalt and predominates the $\mathrm{MO}$ properties of the media.

In Figs. 11 (TM light incidence) and 12 (TE light incidence), we show MO-FS of the media with $P_{M}=10 \sim 100 \%$. In these figures, the apparent Faraday rotation angle $\theta_{F}(\lambda)$ and the ellipticity $\eta_{F}(\lambda)$ were defined by $\theta_{F}=\theta(L) / L$ and $\eta_{F}=\eta(L) / L$, respectively. A strong correlation between these spectra and the experimental ones are seen: The MO effect is considerably small for the TM light incidence (Figs. 11 and 6), whereas it is very unique for the TE light incidence (Figs.12 and 7). As for the magnitudes of $\theta_{F}$ and $\eta_{F}$, however, the theoretical ones (Fig.12) are about 100 times larger than the experimental ones (Fig.7). This is presumably caused by the difference in the concentration of cobalt. In fact, the effective concentration of cobalt in the actual films of $C_{C D}=75 \%$ was estimated to be $4 \sim 5 \%$ of cobalt from their

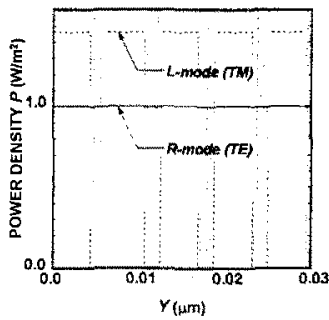

(a) Power density

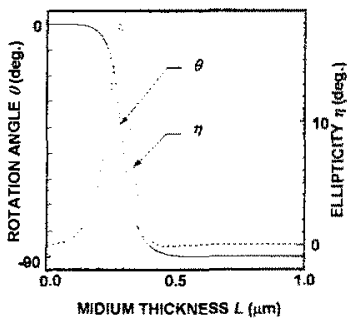

(b) Mode conversion
Fig. 10 Distribution of power density (a) and mode conversion from TE to TM (b) for $P_{M}=30 \%$ and $\lambda=381.5 \mathrm{~nm}$.
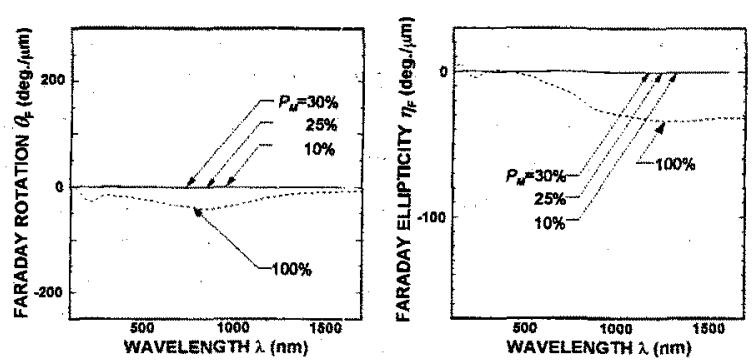

Fig. $11 \mathrm{MO}$-FS of the discrete media for the TM light incidence.
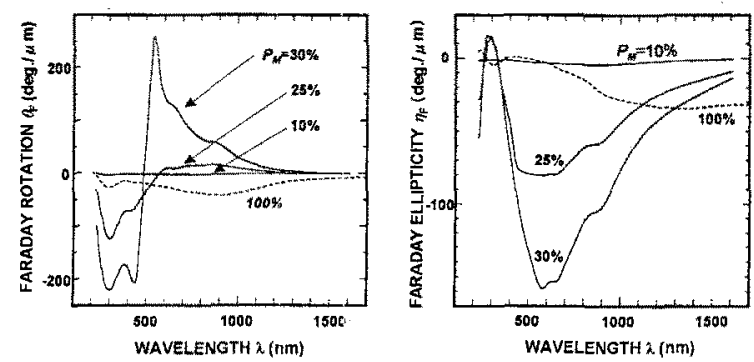

Fig. 12 MO-FS of the discrete media for the TE light incidence.

saturation magnetizations. We notice in Fig. 12 that the shapes of theoretical MO-FS of $P_{M}=30 \%$ are very similar to those of experimental ones of $C_{C O}=30 \%$ in Fig.5. This is, however, considered to be rather contingent, because the films in Fig.5 had no particular treatments for uniaxial orientations, although the possibility of chain-cluster formation of cobalt particles due to another agent still remains.

\section{CONCLUSION}

The present results showed that the uniaxial orientation of cobalt clusters in a transparent matrix yielded the polarizing effect with very unique MO-FS which were entirely different from those of continuous films. In general, the MO-FS of the films are very sensitive to the uniaxial geometry of film structures, suggesting, in turn, that the active design in $\mathrm{MO}-$ FS can be achieved by using, the discrete films with uniaxial orientations. To confirm these results more quantitatively, further experimental and theoretical works are now under progress.

\section{REFERENCES}

[1] K. Matsumoto, S. Sasaki, K. Haraga, K. Yamaguchi, and T. Fujii; J. Appl. Phys., vol.73 (1993) 339.

[2] A. Horikawa, K. Yamaguchi, M. Inoue, and T. Fujii, Proc. Intern. Conf. on Nanocrystals and Granular Mat., (1996) in press.

[3] M. Inoue, T. Yamamoto, K. Isamoto, and T. Fujii; J. Appl. Phys., Proc. MMM'95, vol.79 (1996) in press.

[4] M. Inoue, K. Isamoto, T. Yamamoto, and T. Fujii; J. Appl. Phys., vol.79 (1996) 1611.

[5] P. B. Johnson and R. W Christy; Phys. Rev. B, vol.9 (1974) 5056.

[6] G. S. Krinchik and V. A. Artem'ev; Soviet Physics JETP, vol.26 (1968) 1080 . 